\title{
Muscle Spindle Formation and Differentiation in Regenerating Rat Muscle Grafts
}

\author{
SHFRRY I. ROGFRS ${ }^{1}$ \\ Departments of Anatomy and Biological Sciences, University of Michigan, Ann Arbor, Michigan 48109
}

Received February 16, 1982; accepted in revised form July 26, 1982

\begin{abstract}
Muscle spindle development and function are dependent upon sensory innervation. During muscle regeneration, both neural and muscular components of spindles degenerate and it is not known whether reinnervation of a regenerating muscle results in reestablishment of proper neuromuscular relationships within spindles or whether sensory neurons may exert an influence upon differentiation of these spindles. Muscle spindle regeneration was studied in bupivacainetreated grafts of rat extensor digitorum longus (EDL) muscles. Three types of EDL graft were performed in order to manipulate the extent to which regenerating spindles might be reinnervated: (1) grafts reinnervated following severance of their nerve supply (standard grafts); (2) grafts in which intact nerve sheaths appear to facilitate reinnervation (nerveintact grafts); and (3) grafts in which reinnervation was prevented (nonreinnervated grafts). Complete degeneration of muscle fibers occurred in all grafts prior to regeneration. Initial formation of spindles in regenerating EDL grafts is independent of innervation; intrafusal muscle fibers degenerate and regenerate within spindle capsules that remain intact and viable. The extent of spindle differentiation was evaluated in each type of graft using criteria that included nucleation and ATPase activity, both of which have been shown to be regulated by sensory innervation, as well as the number of muscle fibers/spindle and morphology of spindle capsules.

While most spindles contained normal numbers of muscle fibers, most of these fibers were morphologically and histochemically abnormal. Alterations of ATPase activity occurred in all spindles, but were least severe in nerve-intact grafts. While fully differentiated nuclear bag and chain fibers were not observed in regenerated spindles, large, vesicular nuclei, similar to those of normal intrafusal fibers, were present in a small number of spindles in nerve intact grafts. Sensory nerve terminations were observed only in those spindles that also contained the distinctive nuclei. This study suggests that a specific neurotrophic influence is necessary for regeneration of normal intrafusal muscle fibers and that this influence corresponds to the properly timed sensory neuron-muscle interaction which directs muscle spindle embryogenesis. However, the infrequent occurrence of characteristics unique to intrafusal muscle fibers indicates that reinnervation of regenerating muscle grafts by sensory neurons is inadequate and/or faulty.
\end{abstract}

\section{INTRODUCTION}

Muscle spindles are encapsulated sensory receptors which function in the monosynaptic stretch reflex and in the regulation of muscle tone. Major components of spindles are highly specialized muscle fibers, the intrafusal fibers, which acquire their unique characteristics under the influence of sensory neurons (Milburn, 1973; Zelená, 1957). This influence begins prenatally, as sensory axons induce spindle embryogenesis, and it is required for maintenance of intrafusal fiber specializations in the adult (Tower, 1932; Schröder, 1974a,b; Schröder et al., 1979). Most prominent among the morphological characteristics which distinguish intrafusal from extrafusal muscle fibers are their small size and their large, vesicular nuclei which are arranged in bags (nuclear bag fibers) and chains (nuclear chain fibers) at the equator of the spindles. The myofibrillar ATPase activity of intrafusal fibers also differs from that of ex-

\footnotetext{
${ }^{1}$ Present address: Department of Anatomy, 4-135 Jackson Hall, University of Minnesota Medical School, Minneapolis, Minn. 55455.
}

trafusal fibers and appears to reflect the influence of their sensory and/or motor innervation (Kucera, 1980b; Zelená and Soukup, 1973).

Muscle spindles form in regenerating mammalian muscles (Carlson and Gutmann, 1975; Hakelius, 1977; Schmalbruch, 1977) but little is known about the extent of differentiation of spindles in these muscles or about specific factors that influence this process (Kucera, 1980a; Milburn, 1976). In an earlier study (Rogers and Carlson, 1981), we found that formation of spindles in regenerating rat muscles does not require sensory innervation and therefore differs from spindle embryogenesis in this respect. However, a specific neurotrophic influence during regeneration may be critical to restoration of the unique morphology of intrafusal fibers. Most studies concerning regulation of intrafusal fiber structure and function have focused upon damage to the nerve supply of developing (Werner, 1973; Zelená and Soukup, 1973-1975) and adult (Ip, et al., 1977; Schröder, $1974 a, b)$ muscles. While neural regeneration and reinnervation of spindles were evaluated in these studies, 

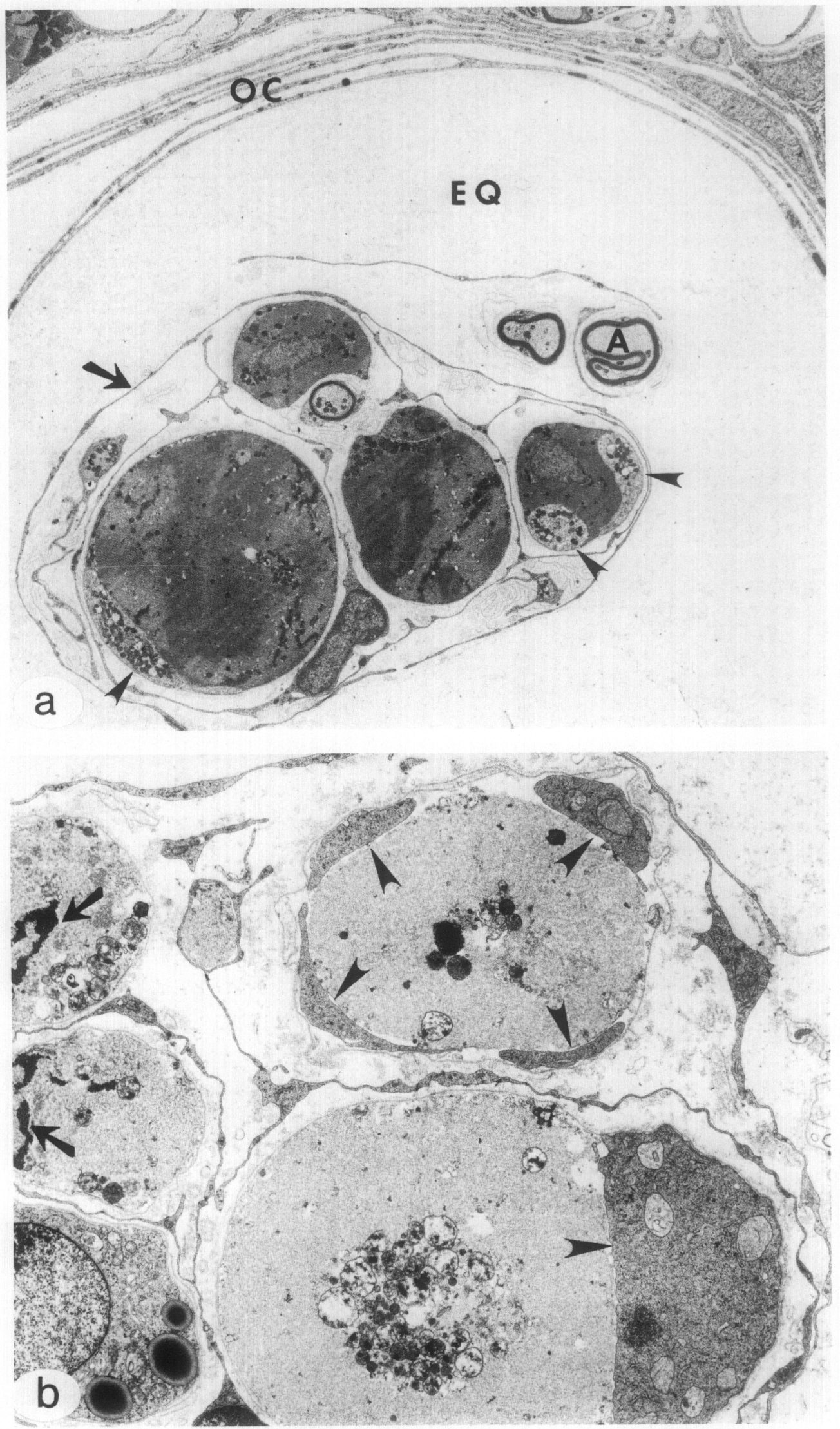
intrafusal muscle fibers did not undergo concomitant regeneration. Milburn (1976) damaged both neural and muscular components of spindles by injecting bupivacaine, a myotoxic local anesthetic, into intact rat muscles. This treatment results in areas of muscle fiber regeneration, but the initial damage and sites of subsequent degeneration are focal and nonuniform, making results difficult to interpret.

Clarification of the processes involved in spindle formation in regenerating muscle will aid in understanding neuromuscular interactions in general and differentiation of muscle grafts in particular. First, while motor reinnervation of regenerating muscles has been studied (Bader, 1980; Carlson et al., 1979, 1981), the ability of sensory neurons to return to appropriate target sites in these muscles has not been evaluated. Second, although the influence of sensory neurons upon embryological development of intrafusal fibers is clear, neurotrophic requirments during spindle regeneration are not understood. Finally, appropriate sensory neuron-muscle interactions are critical to complete functional differentiation of regenerating muscles. Particularly when clinical applications of muscle grafting are considered (Hakelius, 1977, 1979; Thompson, 1979), it is important to evaluate the variables that may be involved in return of the sensory feedback normally provided by muscle spindles.

The experiments presented here were designed to (1) document the process by which spindles form in regenerating muscle grafts; (2) evaluate neural influences upon the extent to which intrafusal fibers differentiate in these grafts; and (3) relate these assessments to reinnervation of the muscle graft as a whole. Because several studies have documented the morphological and physiological parameters of muscle regeneration in the rat extensor digitorum longus (EDL) muscle (Carlson, 1976; Carlson and Gutmann, 1975; Carlson et al., 1979, 1981), this muscle was used in the present study. In order to ensure that regeneration resulted from extensive, uniform damage to all nerve and muscle fibers, whole muscle grafting was combined with bupivacaine treatment. Spindles that form in regenerating EDL muscle grafts face a severe challenge to their ability to regain normalcy. Not only must damaged nerve fibers regenerate and reestablish peripheral connections, but these connections must be made with muscle cells that are themselves regenerating. By manipulating the extent to which regenerating spindles in these grafts may be reinnervated, the role of nerves in their differentia- tion may be evaluated. The criteria used to assess differentiation of regenerated spindles included number of muscle fibers/spindle, nucleation and ATPase activity of encapsulated muscle fibers, and morphology of the spindle capsule.

\section{MATERIALS AND METHODS}

\section{Surgical Procedures}

Male Sprague-Dawley rats, weighing approximately $200 \mathrm{~g}$ at the time of surgery, were used in this study. Anesthesia was with ether or ether in combination with sodium pentabarbitol $(50 \mathrm{mg} / \mathrm{kg}$ body wt).

The standard technique of grafting has been described in detail by Carlson and Gutmann (1975). This technique and two variations, the nonreinnervated graft and the nerve-intact graft, were performed in order to clarify the influence of innervation upon the extent to which regenerating spindles differentiate. Early postgrafting stages were evaluated primarily in standard grafts. All muscles were treated with the myotoxic local anesthetic bupivacaine (Marcaine; $0.75 \%$ bupivacaine in $0.9 \%$ $\mathrm{NaCl}$, Winthrop Laboratories, N. Y.) during the grafting procedure (Rogers and Carlson, 1981). This treatment causes degeneration of multinucleated cells (Benoit and Belt, 1970; Hall-Craggs, 1974; Libelius et al., 1970) and, in combination with muscle grafting, ensures a pure population of regenerating muscle cells (Carlson, 1976).

Standard grafts. EDI, muscles were removed by severing their tendons of origin and insertion and their neurovascular supply, treated with bupivacaine by injection and soaking, and then reconnected to their tendon stumps by suturing. Reinnervation of standard grafts occurs by regeneration of axons into the muscle from the cut end of the nerve (Carlson et al., 1979).

Nonreinnervated grafts. Neural influence upon differentiation of regenerating spindles was prevented by severing and ligating the sciatic nerve (Rogers and Carlson, 1981). The absence of nerve fibers in regenerated grafts was verified in this study by examination of silverstained sections of distal nerve stumps and of grafted muscles 60 days after grafting.

Nerve-intact grafts. Muscles in this group were treated in a manner similar to that described for standard grafts, except that their nerve supply was left intact to facilitate reinnervation. All large blood vessels supplying the muscles were severed and the small vessels that enter the muscle along the nerve trunks were carefully interrupted with the aid of a dissecting microscope.

FIG. 1. (a) Normal muscle spindle, cross section. A, myelinted axon; EQ, equatorial space; OC, outer spindle capsule; arrow, inner capsule; arrowheads, sensory nerve termination. $\times \mathbf{4 5 0 0}$. (b) Portion of a spindle in a 2-day standard graft. Myofibrils are disorganized and nuclei are pyknotic (arrows). Portions of several possibly myogenic cells (arrowheads) are located beneath the basal laminae of the degenerating muscle fibers. $\times 3900$. 

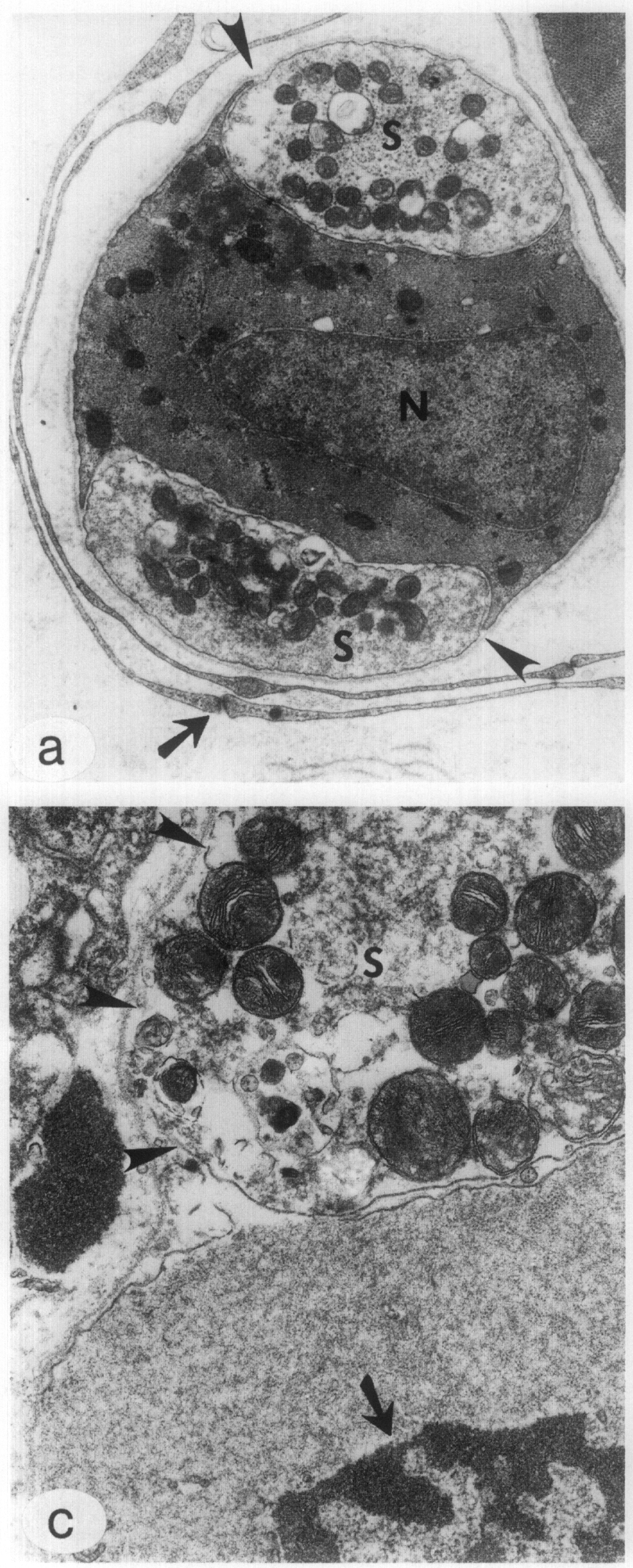
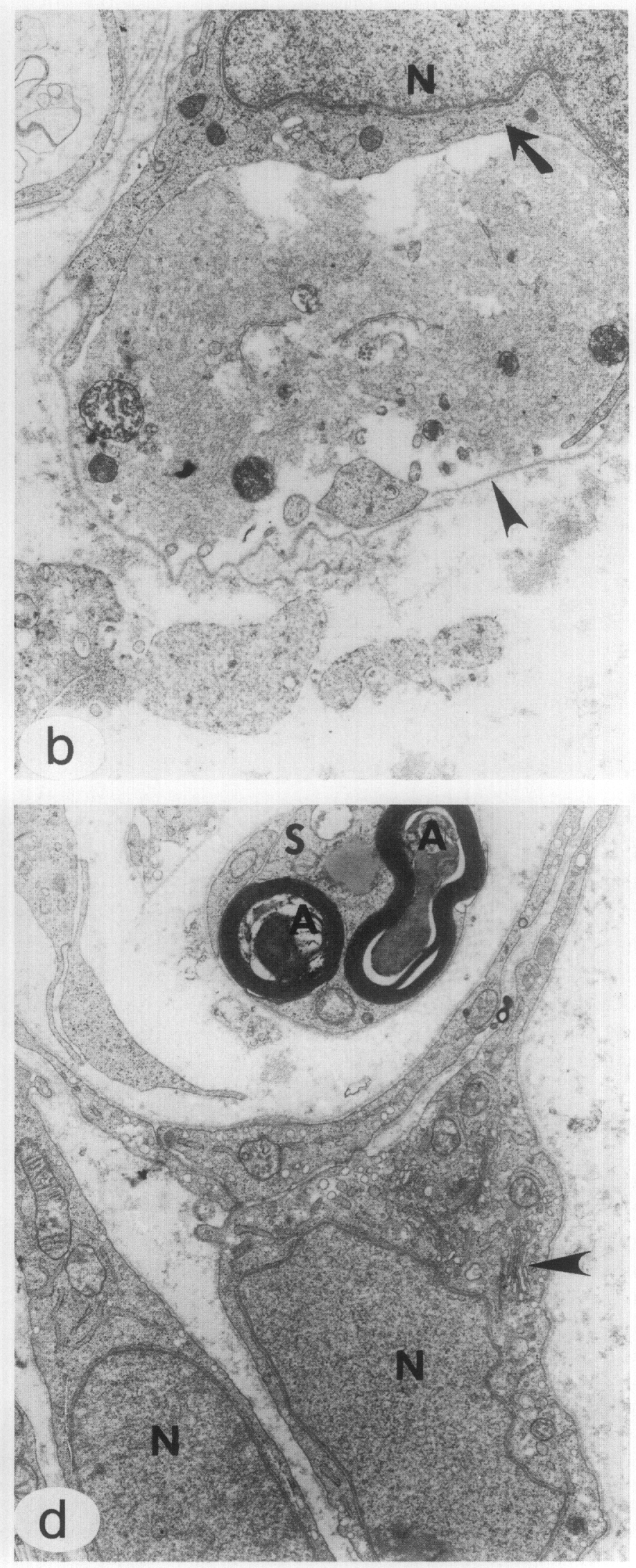
Tendons were cut, the muscles were injected with bupivacaine, and the tendon stumps were then reconnected with sutures. Degeneration of muscle fibers in nerve-intact grafts occurs as in standard grafts and intramuscular portions of nerve fibers also degenerate and regenerate (Carlson et al., 1981).

\section{Processing of Tissue}

The course of spindle degeneration and early regeneration was examined in muscles fixed during the first postoperative week. Characteristics indicative of spindle differentiation were analyzed after 60 days of regeneration, by which time both muscle and nerve components were expected to be stable (Carlson, 1976; Carlson et al., 1979). Spindles in normal EDL muscles served as controls.

Light microscopy. Muscles were fixed in Bouin's fixative, dehydrated through a graded series of alcohols, and embedded in paraffin. Serial sections were cut at $15 \mu \mathrm{m}$ and mounted on glass slides. For morphological observations, sections were stained with hematoxylin and eosin; the presence or absence of nerve fibers was assayed using Hsu's (1974) moditication of the Palmgren silver impregnation technique.

Electron microscopy. Animals were perfused through the abdominal aorta with Karnovsky's fixative. EDL muscles were immersed in the fixative for an additional $12-20 \mathrm{hr}$ and then rinsed in $0.1 \mathrm{M}$ sodium cacodylate buffer (ph 7.4). In order to locate spindles, the muscles were embedded in agar and cut into $200-\mu \mathrm{m}$ cross sections on a Smith-Farquhar TC-2 tissue sectioner. These slices were postfixed in $1 \%$ osmium tetroxide, dehydrated, and embedded in Spurr low-viscosity resin (Polysciences). One-micrometer sections of the slices were examined for spindles and the blocks then trimmed accordingly. Thin sections of the spindles were stained with uranyl acetate and lead citrate and examined under a Philips 400 electron microscope.

Histochemistry. Muscles were quickly removed from the animal and frozen in isopentane cooled with dry ice. Serial frozen sections from several portions of each muscle were cut at 10-12 $\mu \mathrm{m}$. Adjacent sections were placed on alternating slides designated for either acid (ph 4.6) or alkaline ( $\mathrm{pH} 10.4$ ) preincubation prior to the
ATPase reaction (Chayen et al., 1979). When experimental muscles were being stained, sections of normal rat EDL or soleus muscles were placed on each slide to serve as controls for the histochemical reaction.

\section{RESULTS}

\section{The Process of Spindle Formation in Regenerating Muscle}

During the first week after surgery, there are no noticeable histological differences between nerve-intact, standard, and nonreinnervated grafts. In semiserial cross sections examined at the light microscopic level, and in random sections studied with EM, no surviving muscle fibers were observed. Particular attention was directed toward nerve-intact grafts, where the possibility of muscle fiber survival due to persistance of small blood vessles might be greater than that in grafts which are completely removed from the animal during surgery. Carlson et al. (1981) have demonstrated that, when care is taken to sever the small vascular branches that travel with the nerve, the number of surviving fibers in nonbupivacaine-treated grafts does not differ significantly from the number in standard grafts. In the present study, it was consistently observed that, when bupivacaine treatment is combined with the nerve-intact grafting procedure, all multinucleated fibers are destroyed.

The course of degeneration and early regeneration of extrafusal muscle fibers has been described by Carlson (1976) and Hansen-Smith and Carlson (1979) and was not found to differ among the types of grafts used in this study. In brief, the ischemic, bupivacaine-treated muscle fibers began to degenerate during the first postoperative day. Fragmentation of cell membranes and disruption of organelles were followed, a few days later, by phagocytosis by cells which presumably migrate out of ingrowing blood vessels. This cell-mediated destruction takes place within the basal laminae of the muscle fibers, which remain largely intact throughout this stage as well as during subsequent muscle fiber regeneration. Proliferation of myoblasts, derived from muscle satellite cells (Snow, 1978), and their fusion into myotubes, was completed within the first week after grafting.

In an earlier study (Rogers and Carlson, 1981), it was

FIG. 2. (a) Intrafusal fiber in a normal rat EDL muscle spindle contains a large nucleus (N) and two large sensory nerve terminals (S). A common basal lamina (arrowheads) invests both the muscle fiber and the nerve endings which spiral around it. Arrow, inner capsule cell processes. $\times 16,000$. (b) Extensive internal degeneration of an intrafusal fiber in a 4-day standard graft. A large, viable cell (arrow) lies beneath the basal lamina (arrowhead) of the degenerating fiber. Synthetic activity of the viable cell is suggested by the large euchromatic nucleus (N) and abundance of ribosomes. $\times 11,800$. (c) Degenerating sensory nerve terminal (S) of an intrafusal fiber in a 2-day nerve-intact graft. Portions of the axolemma are disrupted or have disappeared entirely (arrowheads) although the basal lamina is intact. Degenerating sarcoplasm is flocculent in appearance and the nucleus (arrow) is pyknotic. $\times 28,800$. (d) Inner capsule cells in a 2-day graft. Synthetic and transport activity is suggested by the euchromatic nucleus (N), Golgi apparatus (arrowhead), rough endoplasmic reticulum, free ribosomes, and numerous vesicles. A Schwann cell (S) surrounds degenerating axons (A) and their disrupted myelin sheaths. $\times 11,700$. 


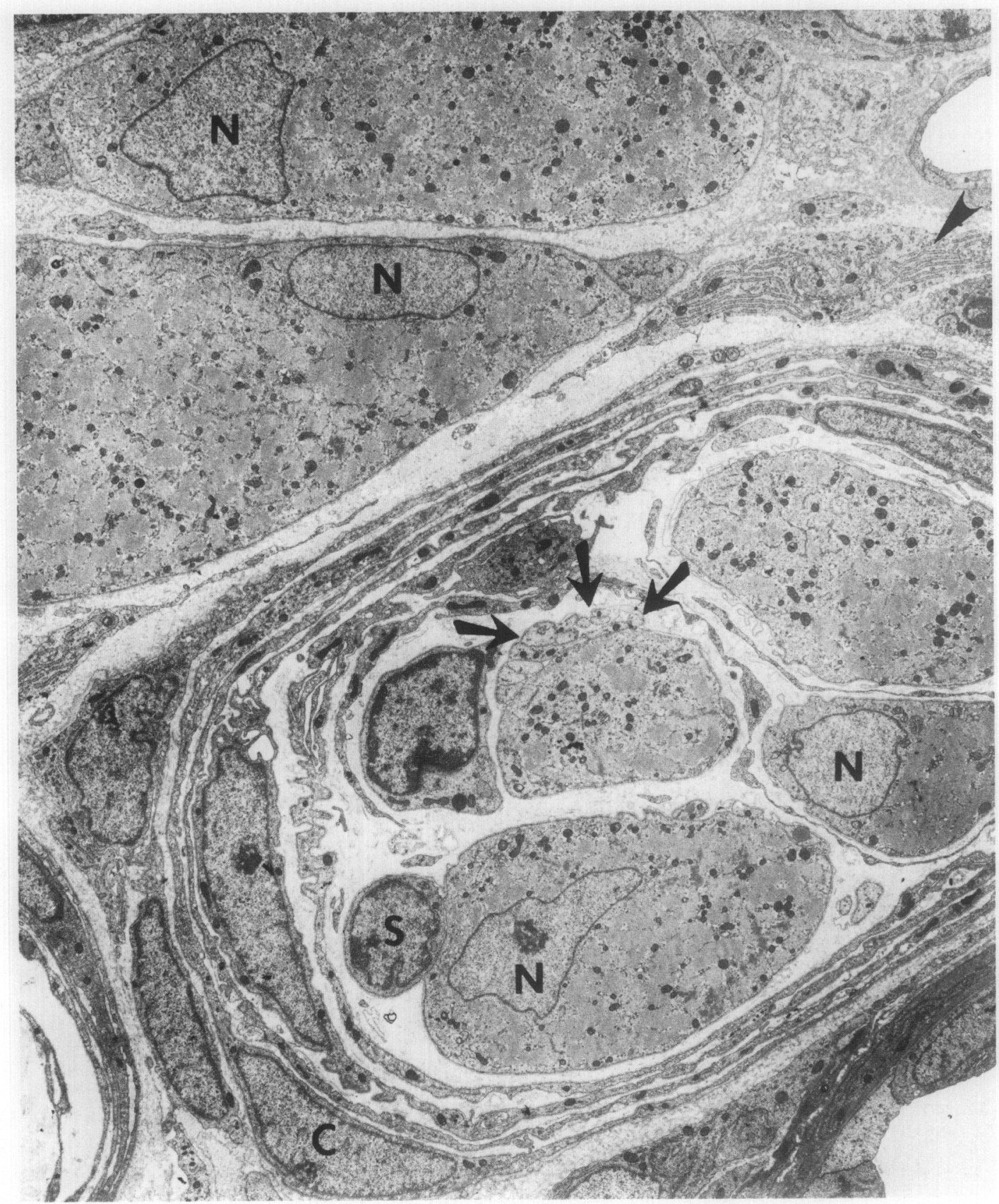

FIG. 3. Regenerating intrafusal and extrafusal muscle fibers in a 6-day standard graft. Myonuclei (N) are euchromatic and a satellite cell (S) is located under the basal lamina of one encapsulated fiber. Portions of unidentified cells (arrows) are associated with one of the encapsulated fibers; they contain mitochondria and other organelles but do not appear to be neuronal or myogenic. Such structures were also observed in association with extrafusal muscle fibers. $\mathrm{C}$, nucleus of spindle capsule cell; arrowhead, fibroblast. $\times \mathbf{4 4 0 0}$.

reported that intrafusal muscle fiber degeneration parallels that of the extrafusal fibers. In the present study, light and electron microscopic observations did not reveal any surviving intrafusal fibers in any of the grafts. Again, nerve-intact grafts were examined with particular care. Distinct degenerative changes in intrafusal fibers in all types of graft could be seen by 2 days after grafting. In comparison with the morphology of normal intrafusal fibers (Figs. 1a and 2a), myofibrils were disorganized and cellular organelles were fragmented and clumped together (Fig. 1b). Nuclei of these fibers were often pyknotic and mitochondria were swollen or disrupted. Within the intact basal laminae of many degenerating fibers, viable active cells were present (Figs. 
TABLE 1

The Extent of Differentiation of Regenerated Muscle Spindles

\begin{tabular}{|c|c|c|c|c|c|c|}
\hline \multirow[b]{2}{*}{ Spindles in } & \multirow{2}{*}{$\begin{array}{c}\text { Average No. } \\
\text { of fibers }\end{array}$} & \multirow[b]{2}{*}{ Bags and chains } & \multirow{2}{*}{$\begin{array}{l}\text { Equatorial } \\
\text { space }\end{array}$} & \multirow{2}{*}{$\begin{array}{l}\text { ATPase } \\
\text { profile }{ }^{a} \\
(\%)\end{array}$} & \multicolumn{2}{|c|}{ Innervation } \\
\hline & & & & & Motor & Sensory \\
\hline Standard grafts & $\begin{array}{l}3.6 \pm 1.3(\mathrm{SD}) \\
\quad \text { (range }=2-5 \\
n=20 \text { spindles) }\end{array}$ & $\begin{array}{l}\text { None observed } \\
\quad(n=20 \text { spindles })\end{array}$ & $\begin{array}{l}\text { Present ( } n=20 \\
\quad \text { spindles) }\end{array}$ & 12 & $\begin{array}{l}\text { Present in three } \\
\text { spindle cross } \\
\text { sections } \\
(n=17)\end{array}$ & $\begin{array}{l}\text { None observed } \\
\quad(n=17)\end{array}$ \\
\hline
\end{tabular}

${ }^{a}$ Percentage of spindle cross sections with intrafusal fibers of more than one type. See text for details.

$1 \mathrm{~b}$ and $2 \mathrm{~b}$ ). It is not possible to identify these cells with certainty at this stage, as myogenic and phagocytic cells, which are also seen beneath basal laminae of degenerating muscle cells, may be similar in appearance (Trupin et al., 1979). However, cells such as the one in Fig. 2b contain large, euchromatic nuclei and numerous ribosomes, indicative of synthetic activity, rather than the lysosomes and vacuoles generally associated with active phagocytic cells. Internal degeneration of intrafusal fibers was extensive by 4 days after grafting (Fig. 2b), but the cells which appeared to be myogenic exhibited signs of synthetic activity.

Spindle capsules were present and intact throughout all stages of muscle degeneration and regeneration. Occasional areas of capsule damage were noted, but cellular degeneration was not observed.

By the second day after grafting, degenerating sensory endings contained disorganized mitochondria and scattered debris. Portions of the axolemma were absent, although the basal laminae surrounding these terminals was undamaged (Fig. 2c). Such indications of sensory terminal degeneration were also reported by DeSantis and Norman (1979) after severing or freezing a nerve. All preterminal axons encountered in early grafts also were degenerating (Fig. 2d).

Regeneration of intrafusal muscle fibers was well underway by 6 days after surgery (Fig. 3), and the appearance of the encapsulated fibers was identical to that of the surrounding extrafusal muscle fibers. Their basal laminae were intact and frequently appeared to be "loose" around portions of the fibers. Cells that resemble myogenic satellite cells (Snow, 1978) were associated with many regenerating muscle fibers. Like satellite cells, they were located beneath the muscle fiber basal lamina and had a large nuclear:cytoplasmic ratio.

\section{ThE EXTENT OF DifFerentiation of REGENERATED SPINDLES}

\section{General Morphology}

Spindles in standard and nerve-intact grafts were relatively easy to identify in cross sections. As in normal muscle (Fig. 4a), spindle capsules in these grafts clearly separated the encapsulated group of muscle fibers from the surrounding extrafusal muscle (Figs. $4 \mathrm{~b}$ and c). An inner capsule which was normal in appearance invested most groups of encapsulated fibers in the equatorial region of the spindles (Figs. $4 \mathrm{~b}$ and c). Encapsulated fibers were generally smaller than extrafusal fibers in the same graft, although this size difference was most pronounced in nerve-intact grafts (compare Figs. $4 b$ and c).

Nonreinnervated grafts contained large amounts of connective tissue which obscured some spindles at the light microscopic level; others, such as the one illustrated in Fig. 4d, were relatively distinct. Both encapsulated and extrafusal fibers in nonreinnervated grafts were atrophic and their myofibrils often appeared to be 

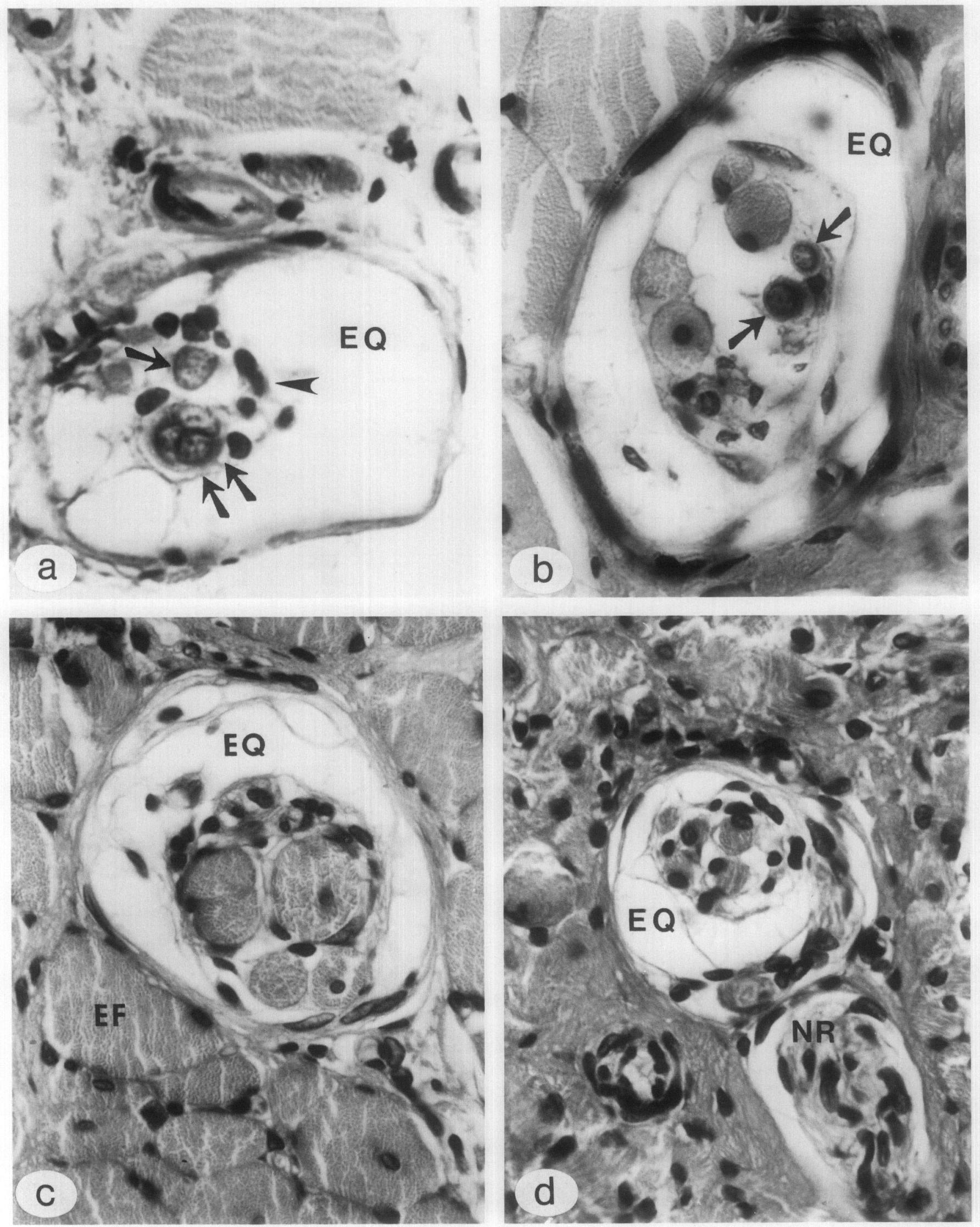
disorganized. Frequently, there was little or no size difference between encapsulated and extrafusal muscle fibers.

\section{Intrafusal Muscle Fibers}

Number. The number of encapsulated fibers was determined in normal EDL muscles and in each type of graft. A total of 20 spincles ( 10 in cach of two muscles) from each group was examined. All spindles contained between two and six muscle fibers (Table 1). The mean number of fibers was greatest in nerve-intact grafts ( 4.2 $\pm 1.2(\mathrm{SD}))$ and nearly identical in standard and nonreinnervated grafts $(3.60 \pm 1.3(\mathrm{SD})$ and $3.73 \pm 0.58$ (SD), respectively). The values conformed closely to the normal average of $3.85 \pm 0.75$ (SD) intrafusal fibers.

Morphology. Spindles which were used to determine fiber numbers (see above) were also examined for the presence of nuclear bag and chain fibers. Serial sections of these spindles were traced from pole to pole. Normally, spindles in rat EDL muscles contain two hag and chain fibers. While many regenerated intrafusal fibers contained centrally located nuclei, the majority of these nuclei were small and darkly staining with hematoxylin and eosin, like those of extrafusal fibers (Figs. $4 \mathrm{~b}-\mathrm{d}$ ). Nuclei which form the bags and chains of normal intrafusal fibers are large and vesicular and are often surrounded by only a thin rim of cytoplasm (Fig. 4a). Therefore, only nuclei with this appearance were identified as part of a regenerated bag or chain fiber.

In nerve-intact grafts, 78 intrafusal fibers were examined with the electron microscope (Table 1). Seven fibers contained one or more large, vesicular nuclei similar to those of normal intrafusal muscle fibers. Four of these fibers resembled very abbreviated nuclear chain fibers (Fig. 4b). Three fibers had large, vesicular nuclei that were closely apposed but were not all resolvable within single cross sections as are those of normal nuclear bag fibers (Fig. 4a). While these fibers may have been abbreviated bag fibers, this cannot be stated with certainty. An additional five fibers in nerve-intact grafts contained nuclei that were more difficult to evaluate: they were large and more lightly staining than typical intrafusal nuclei, yet they were not as distinctive in ap- pearance as the fibers illustrated in Fig. $4 \mathrm{~b}$. Therefore, typical intrafusal nuclei were present in at least $9 \%$, and possibly as many as $15 \%$, of regenerated fibers in spindles of nerve-intact grafts. No nuclear bag or chain fibers were observed in either standard or nonreinnervated grafts (Table 1).

Electron microscopic examination of cross sections through 10 spindles in five nerve-intact grafts confirmed the morphology of the intrafusal nuclei described above. Because of potential variation among regenerated spindles, we felt that random sampling would provide more information than would extensive examination of only a few spindles. Large, euchromatic nuclei were present in four fibers distributed between two spindles in two different grafts (Figs. 5a and c). Other such nuclei may have been missed in other spindles, due to the random nature of the sampling process. However, it is significant that when these nuclei were observed, they were associated with sensory nerve terminations (discussed further below); conversely, sensory terminations were not observed in any other sections.

Some muscle fibers in spindles in which sensory innervation was observed exhibited two prominent morphologic characteristics in addition to their nucleation. Two of these fibers contained centrally located accumulations of cytoplasmic organelles (Fig. $5 b$ ). Such accumulations are also located near equatorial nuclei of normal muscle spindles (Ovalle, 1972a). Three of the fibers contained membrane-bound cytoplasmic bodies filled with dense, granular material. One of these structures was closely associated with a small mitochondrion (Fig. 5c), resembling complexes noted by Ovalle (1971). The remaining encapsulated fibers in nerve-intact grafts (those that did not receive sensory innervation) were morphologically similar to the surrounding extrafusal muscle fibers.

Sections of 17 spindles in five standard grafts were examined with the electron microscope. In overall appearance they resembled spindles in nerve-intact grafts which were not associated with sensory nerve terminations. Myofibrillar organization of the encapsulated fibers was identical to that of extrafusal muscle fibers (Figs. 6a and $\mathrm{b}$ ) and all nuclei were heterochromatic (Fig. 6b).

FIG. 4. (a) Cross section through the equatorial region of a spindle in an adult EDL muscle. Large, vesicular nuclei are contained in a nuclear bag (double arrows) and a nuclear chain (single arrow) fiber. Two other intrafusal fibers do not have visible nuclei in this section. The dense, darkly staining nuclei (arrowhead) belong to cells of the inner spindle capsule. EQ, equatorial space. $\mathrm{H}$ and $\mathrm{E}$. $\times 900$. (b) Two small muscle fibers (arrows) in a 60-day nerve-intact graft contain large, vesicular nuclei surrounded by a thin rim of cytoplasm and were identified as nuclear chain fibers. The nuclei of these fibers can be distinguished easily from those of the other muscle fibers in this spindle. This group of encapsulated muscle fibers is surrounded by a large equatorial space (EQ) and an inner capsule. $\mathrm{H}$ and $\mathrm{E}$. $\times 900$. (c) A typical spindle in a 30-day standard graft. The encapsulated muscle fibers are morphologically similar to the extrafusal fibers (EF) although somewhat smaller. A space (EQ) is present between the bundles of muscle fibers and the spindle capsule. $\mathrm{H}$ and E. $\times 900$. (d) A spindle in a 30 -day nonreinnervated graft contains small muscle fibers with relatively large nuclei. The extrafusal muscle is disorganized and is surrounded by a large amount of connective tissue. A nerve remnant (NR) is adjacent to the spindle. $\mathrm{EQ}$, equatorial space. $\mathrm{H}$ and $\mathrm{E}$. $\times 900$. 

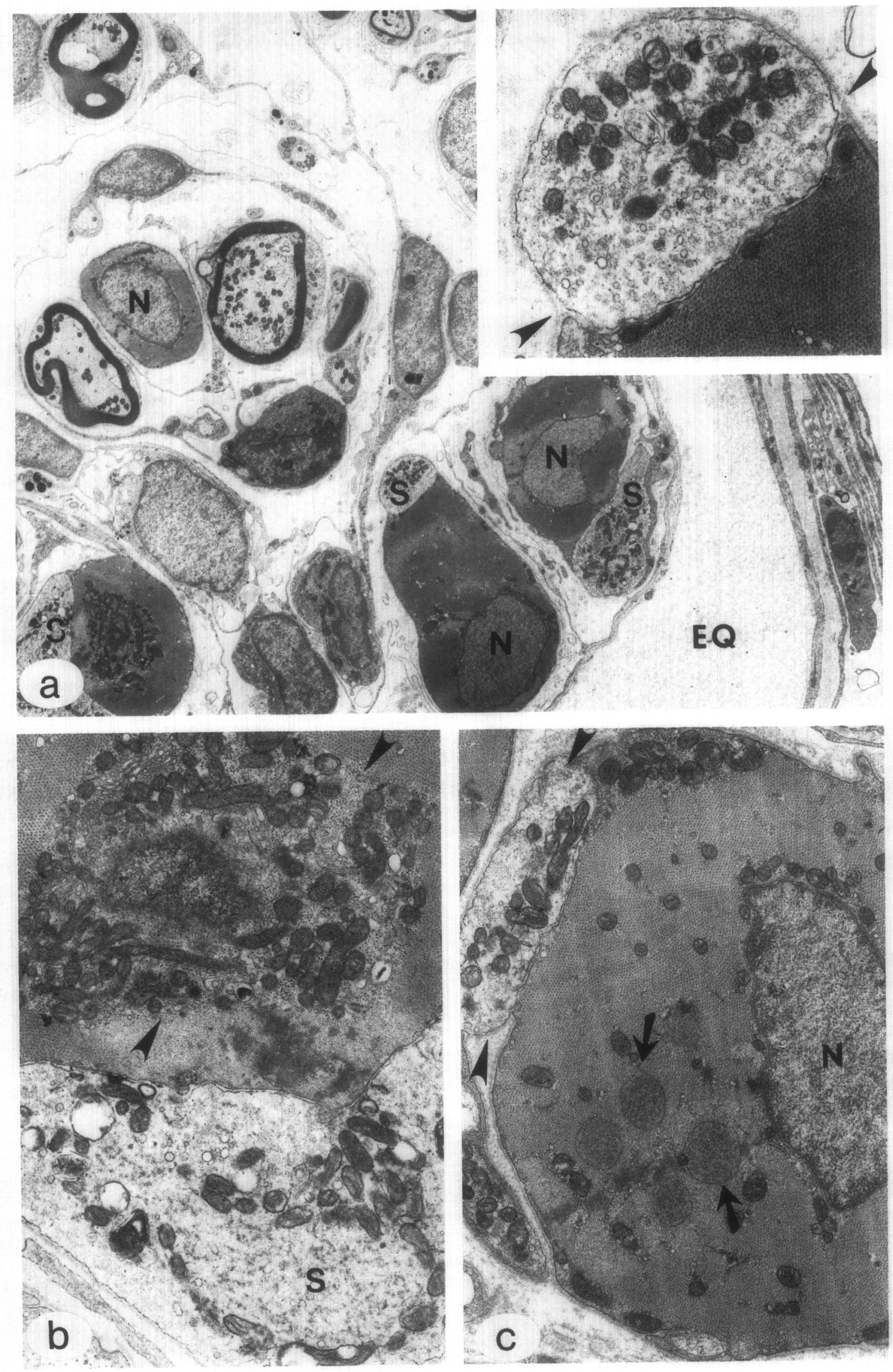
Muscle fibers in spindles of nonreinnervated grafts were highly variable in terms of size and ultrastructure. In the cross sections examined (15 spindles in five grafts), encapsulated and extrafusal fibers were approximately the same size (Fig. 6c). Both types of fibers exhibited varying degrees of abnormality. While some fibers, like those in Fig. 6c, appeared to be relatively healthy, myofibrillar organization in others was extremely disrupted. Nuclei of many fibers, both within and outside of spindle capsules, were euchromatic, lobulated, and often contained prominent nucleoli (Fig. 6c). Nonreinnervated grafts tended to contain excessive amounts of collagen surrounding the muscle fibers.

It is noteworthy that spindles in all types of graft contained capillaries (Figs. 6 and 8a). Normally, capillaries are not found within the periaxial space of rate muscle spindles (James and Meek, 1971; Merrilees, 1960).

Histochemistry. Normal rat EDL muscles are composed predominantly of fast extrafusal fibers. These fibers stain intensely when the ATPase reaction is preceded by alkaline preincubation and lightly following acid preincubation. Scattered slow extrafusal muscle fibers react in an opposite manner, exhibiting ATPase stability at a low $\mathrm{pH}$.

Normal intrafusal muscle fibers have a much more complex ATPase profile, which probably reflects their unique innervation. Each spindle contains two or three types of fibers, all of which do not exhibit the dichotomy of $\mathrm{pH}$-dependent staining that is typical of extrafusal muscle fibers. In addition, single intrafusal fibers may have different staining characteristics along their length (see Kucera et al. (1978) for a more detailed description of muscle spindle histochemistry). No attempt was made in the present study to document details of the histochemistry of the normal muscle spindle. Rather, the ATPase reaction was used to evaluate two of the criteria which appear to be indicative of the unique innervation of intrafusal muscle fibers (Kucera et al., 1978): (1) the presence of two or three fiber types in each spindle, in groupings which distinguish them from the surrounding extrafusal fibers; and (2) both alkaline and acid stability of at least one fiber per spindle.

All spindles in normal EDL muscles contained both light and dark fibers after staining for alkaline-stable ATPase. Staining patterns varied somewhat among these sections, but this probably reflects a difference in their level within a spindle (Kucera et al., 1978). The spindle shown in Fig. 7a and b contains a complement of three intrafusal fiber types: the large "bag 2" fiber which is both acid and alkaline stable; one "bag 1" fiber, which reacts more intensely at low than at high $\mathrm{pH}$; and four "chain" fibers which, in this section, do not exhibit the extreme reaction of the "fast" extrafusal fibers but do tend toward an alkaline-stable profile when compared to the "slow" extrafusal fibers in the same section (not shown).

In nerve-intact grafts, approximately half of the spindles contained both light and dark fibers after alkaline preincubation (Table 1; Fig. 7c; 41 spindles in six grafts were examined). The number of dark vs light fibers per spindle varied, but the majority of these fibers reversed their ATPase profile following acid preincubation (Figs. $7 \mathrm{c}$ and d). Occasionally, fiber type differences that were apparent after alkaline preincubation disappeared after preincubation at a low $\mathrm{pH}$, with all fibers then staining uniformily. The remaining spindles had an ATPase profile identical to that of the surrounding extrafusal fibers: dark at an alkaline $\mathrm{pH}$ and light at an acid $\mathrm{pH}$ (Figs. $7 \mathrm{c}$ and $\mathrm{d}$ ). Scattered patches of extrafusal fibers of opposite type were found in most nerve-intact grafts.

Of the 33 spindles in standard grafts that were studied, approximately $12 \%$ contained both light and dark fibers (Table 1). This $12 \%$ was concentrated in 4 of 10 standard grafts. Staining heterogeneity among these fibers was often less distinct than that in nerve-intact grafts. Most intrafusal fibers in standard grafts stained with an intensity which was identical to that of the surrounding extrafusal fibers (Fig. 7e). The ATPase profile of extrafusal fibers in these grafts was largely homogeneous, with occassional patches of fibers of opposite type.

In nonreinnervated grafts, muscle fibers in all 41 spindles examined had an ATPase profile identical to that of the extrafusal fibers. Staining of each graft was homogeneous (Fig. 7f) and was usually less intense than was staining of standard and nerve-intact grafts.

\section{Spindle Capsules}

By following serial sections of spindles, it was clear that capsules in all grafts maintained their distinctive shape. The capsules expanded around the central portions of the enclosed muscle fibers, forming the equa-

FIG. 5. (a) A portion of a spindle in a 60-day nerve-intact graft. Three encapsulated fibers contain sensory nerve terminations (S) and two of these fibers, plus a third fiber, have large euchromatic nuclei $(\mathrm{N})$. EQ, equatorial space. $\times 3900$. Inset: Enlargement of one sensory termination. Arrowheads, basal lamina. Note scattered vesicles within the nerve terminal. $\times 25,000$. (b) Enlargement of fiber in the spindle illustrated in a. S, sensory termination. Arrowheads denote accumulation of cytoplasmic organelles. $\times 21,750$. (c) Encapsulated fiber in another 60 -day nerveintact graft. Sensory nerve termination is covered by basal lamina (arrowheads). N, large, euchromatic nucleus; arrows, dense cytoplasmic bodies. $\times 14,000$. 

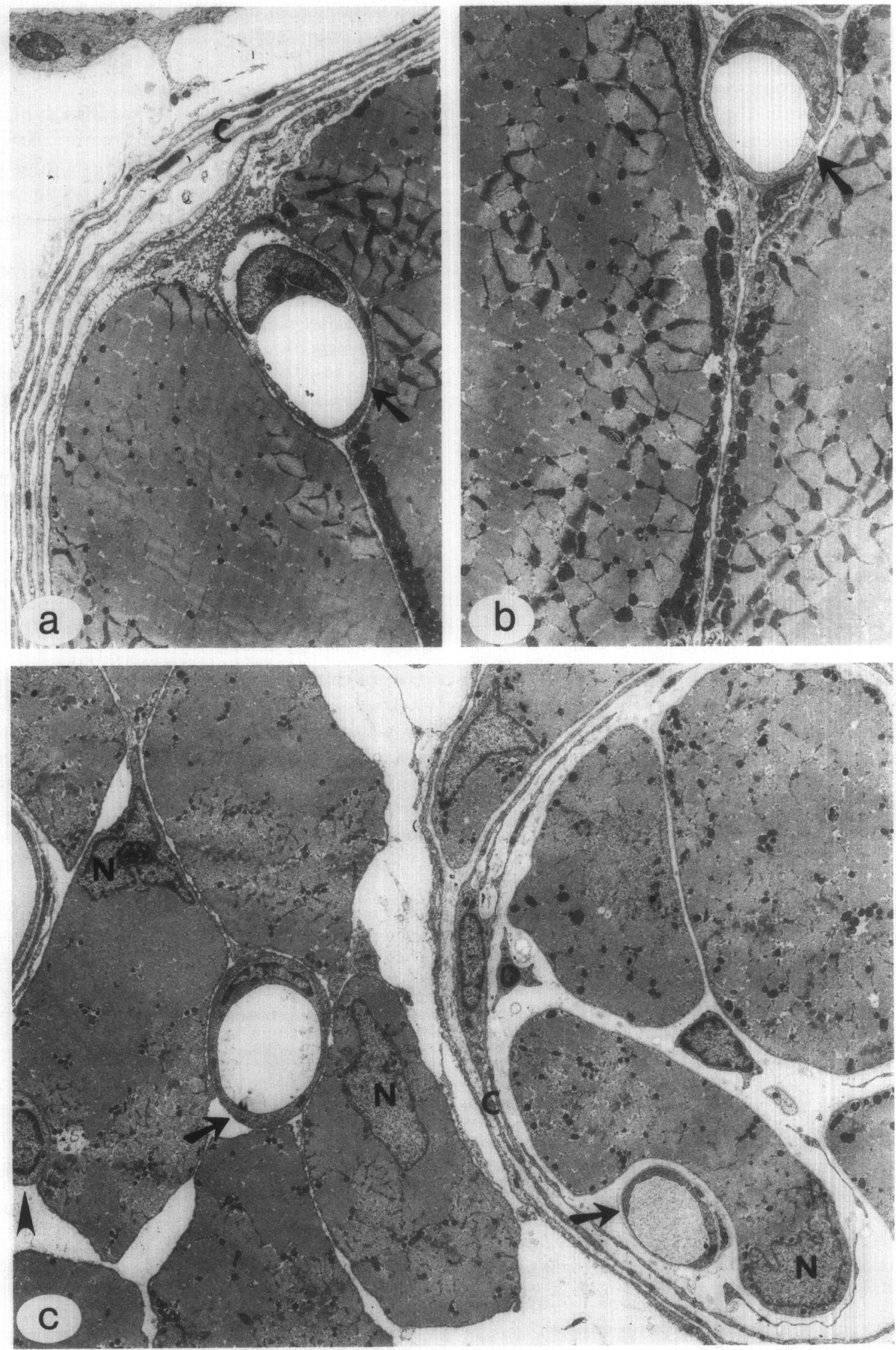
torial space (Fig. 4, Table 1). Normally, this space is believed to be filled with a mucopolysaccharide gel (Brezezinski, 1961; James, 1971) but it is not yet known whether the same substance is present in regenerated spindles.

In all of the EM sections examined, spindle capsules were intact and viable. As is true of normal spindles, the number of capsular layers and their configuration varied among specimens; these variables are also affected by the region of the spindle through which a section is taken. Often, however, processes of inner capsule cells did not surround individual intrafusal fibers as distinctly as they normally do (compare Figs. 1 and 5a). The ultrastructural characteristics of the capsule cells were normal: nuclei tended to be elongated and euchromatic and the cells appeared to be involved in synthetic and/or transport activities.

There was no evidence to suggest formation of new spindle capsules.

\section{Innervation of Regenerated Spindles}

In silver-stained, serial sections of standard and nerve-intact grafts, nerve fibers were associated with all spindles encountered (see Fig. 3, Rogers and Carlson (1981)). However, it could not be determined, at the light microscopic level, whether these fibers terminated or whether they were sensory or motor in nature. Nerve fibers were not observed in serial sections of non-reinnervated grafts.

With the electron microscope, both myelinated and nonmyelinated preterminal axons were observed in spindles of reinnervated grafts. They were located within nerve bundles associated with the spindles (Fig. 8a) and in close proximity to the encapsulated muscle fibers. However, their occurrence was less frequent in standard than in nerve-intact grafts.

Motor nerve terminations and preterminal axons were seen, although infrequently, in standard and nerveintact grafts (Table 1). Two or more motor endings upon a single intrafusal fiber, such as those illustrated in Fig. $8 \mathrm{~b}$, were observed occasionally in both types of graft. Normal intrafusal muscle fibers may also have several motor endplates at their poles (Ovalle, 1972b). Where postjunctional specializations were present, endplate morphology was similar to that of extrafusal muscle in terms of postjunctional folds and vesicle accumulation (Fig. 8c). In one nerve-intact spindle (Fig. 8a), motor axons lying close to two intrafusal fibers were relatively large and were not associated with postjunctional folds. They may have been preterminal axons or terminations similar to trail endings in normal spindles (Ovalle, $1972 \mathrm{~b}$ ). It must be noted, however, that it could not be determined whether the motor axons present were from alpha or gamma motoneurons.

Sensory nerve terminations were observed only in nerve-intact grafts. They were present in 2 of the 10 spindles examined ultrastructurally and were morphologically normal. Mitochondria, filamentous material, and scattered vesicles were contained in the terminals and a common basal lamina enclosed each terminal and its associated muscle fiber.

\section{DISCUSSION}

The major findings of this study are that (1) capsules of muscle spindles remain intact and viable during regeneration of muscle grafts rather than being formed de novo; (2) degeneration and regeneration of intrafusal muscle fibers follow patterns that have been shown to be involved in regeneration of extrafusal muscle fibers; (3) intrafusal muscle fibers may regenerate without sensory innervation, but its absence results in morphological and histochemical abnormalities; and (4) reinnervation of regenerating muscle grafts does not result in fully differentiated muscle spindles. These findings substantiate the results of most previous studies on muscle spindle regeneration and reinnervation, extend analyses of spindle embryogenesis to regeneration in adult muscle, and provide a basis for assessment of sensory reinnervation of regenerating muscle.

\section{SPINDLE Formation IN REgenerating Muscle}

Analysis of early muscle grafts confirms suggestions of previous studies (Rogers and Carlson, 1981; Milburn, 1976) that muscle spindle formation in regenerating mammalian muscles is dependent upon survival of spindle capsules and regeneration of muscle fibers within these capsules and that, therefore, spindles do not regenerate de novo. De novo formation of spindles in regenerating muscle, involving a recapitulation of their embryonic development (Milburn, 1973), would require precisely timed contact between regenerating sensory axons and differentiating myotubes. While such contact is unlikely in standard reinnervated grafts (see Rogers and Carlson, 1981), the possibility exists that it could occur in nerve-intact grafts, in which nerve fibers have

FIG. 6. ( $a$ and b) Sixty-day standard graft. Encapsulated (a) and extrafusal (b) muscle fibers are nearly identical in appearance. C, spindle capsule; arrows, capillaries. (a) $\times 5400$; (b) $\times 6100$. (c) Sixty-day nonreinnervated graft. Encapsulated and extrafusal fibers are morphologically similar in terms of their size, myofibrillar organization and nuclei $(N)$. Arrow, capillaries; arrowhead, portion of satellite cell. $\times 5100$. 

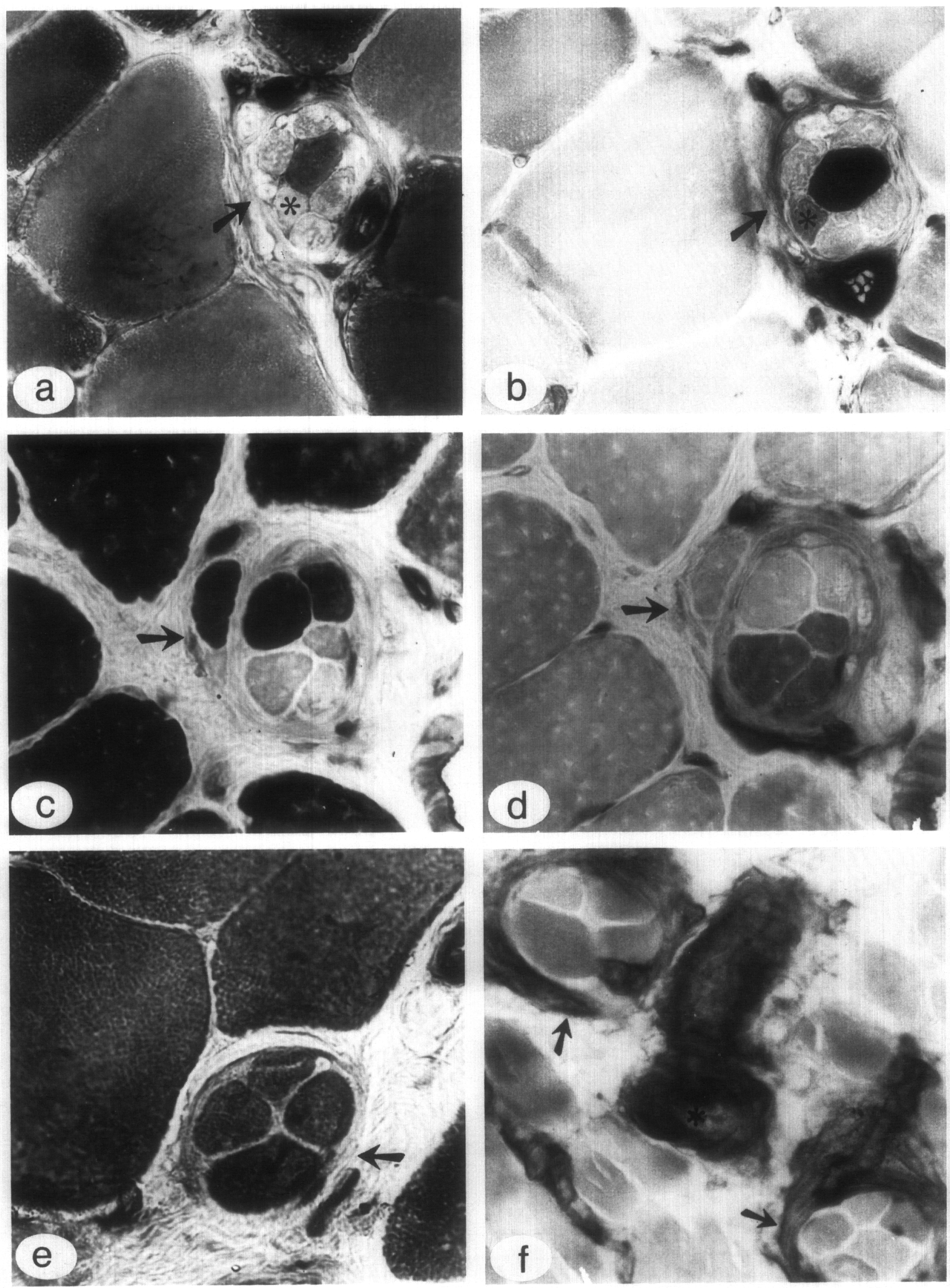
been observed as early as 5 days after surgery (Carlson et al., 1981), when some myotubes might be relatively undifferentiated. However, even in nerve-intact grafts all capsules observed were fully formed throughout all stages of regeneration.

Although de novo formation of spindles appears unlikely in regenerating mammalian muscle, mechanisms of spindle regeneration may vary among species. In birds, spindles appear to form in previously spindleless muscles when transplanted to sites of spindle-containing muscles (Mackenson-Dean et al., 1981). This suygests that spindle regeneration in these muscles is neurally induced and does not require survival of spindle capsules. The contradiction between studies on mammalian and avian spindle regeneration might be due to fundamental class differences in (1) the time course of muscle regeneration and/or (2) nerve-muscle interactions during regeneration. It appears that avian muscle regenerates more slowly than rat muscle (R. S. Hikida, personal communication) which might allow time for interaction between young myotubes and regenerating sensory axons. Further investigation of muscle regeneration in mammals other than rats may reveal a similar phenomenon, particularly where larger size appears to result in a slower course of regeneration (Mufti et al., 1977).

An intriguing question concerning muscle spindle embryogenesis is whether a unique population of myoblasts, destined to become intrafusal fibers, exists during muscle development or whether intrafusal fibers develop from a common pool of myoblasts by virtue of their innervation. Several lines of evidence suggest the validity of the latter alternative. First, in early grafts where innervation is absent, encapsulated and extrafusal fibers are morphologically identical, suggesting uniformity of myogenic cells. These similarities persist unless sensory innervation ensues. Second, MackensonDean et al. (1981) demonstrated that spindles will form from a population of regenerating cells which, during development, form only extrafusal muscle fibers. Third, Elliot and Harriman (1974) showed that intrafusal fibers regenerating without nerves in vitro resemble extrafusal fibers. Although the above studies indicate that intrafusal and extrafusal muscle fibers are not intrinsically different, it is possible that myogenic cells that participate in regeneration do not contain precisely the same information as embryonic myoblasts.

\section{EXTENT OF DifFERENTIATION OF SPINDLES IN REgEnERATED MUSClE}

Morphology. The appearance of large, vesicular nuclei in regenerated intrafusal fibers and the association of these nuclei with sensory nerve terminations strongly suggest a neurotrophic influence similar to that required for differentiation of intrafusal fibers during embryonic development. However, the inadequate differentiation of nuclear bags and chains in regenerated spindles indicates that the extent of neural influence may be markedly reduced. Milburn (1976) reported an absence of nuclear bag and chain fibers in Marcaine-injected, nongrafted muscles and, in an earlier paper (1973), hypothesized that there is a decreasing morphogenetic influence of primary sensory neurons as embryogenesis of spindles progresses. The largest nuclear bag fiber, with the most elaborate nucleation, is the first to form, while sensory terminals do not reach potential chain fibers until several days later. In addition, Milburn points out that regenerating axons in adult animals might exert less influence on their target muscle fibers than do axons in embryonic or neonatal animals. While decreased neural influence may help to explain the faulty differentiation of regenerating intrafusal fibers, it is also possible that the muscle fibers themselves may be unable to respond to such an influence. Particularly in standard grafts, where reinnervation occurs somewhat more slowly than in nerve-intact grafts (Carlson et al., 1981), regenerating myotubes may be fairly well developed by the time that they are innervated. Finally, a likely explanation for faulty differentiation of many intrafusal fibers is that sensory axons only rarely make accurate connections in regenerating muscle grafts.

Two ultrastructural features noted in regenerated spindles with sensory nerve terminations have been described by Ovalle $(1971,1972 b)$ in normal rat intrafusal muscle fibers. Centrally located accumulations of organelles typically occur between equatorial nuclei in normal spindles. The membrane-bound cytoplasmic bodies in regenerated fibers resembled the dilated cisternae of the sarcoplasmic reticulum reported by Ovalle in both equatorial and polar regions. The functional significance of these structures is not clear, but they are associated with sensory innervation in normal and regenerated spindles.

The consistent presence of capillaries in regenerated spindles was surprising, as they are not found in normal

FIG. 7. (a and b) Normal EDL. ATPase reaction following alkaline (a) and acid (b) preincubation. A spindle is indicated by arrows. ${ }^{*}$, small fiber which appears to be a bag 1 fiber. $\times 390$. (c) Sixty-day nerve intact graft, alkaline preincubation. Arrow, spindle. $\times 480$. (d) Section adjacent to $\mathrm{c}$, acid preincubation. $\times 480$. (e) Sixty-day standard graft, alkaline preincubation. $\times 600$. (f) Sixty-day nonreinnervated graft, alkaline preincubation. Arrows, spindles; *, blood vessel. $\times 600$. 

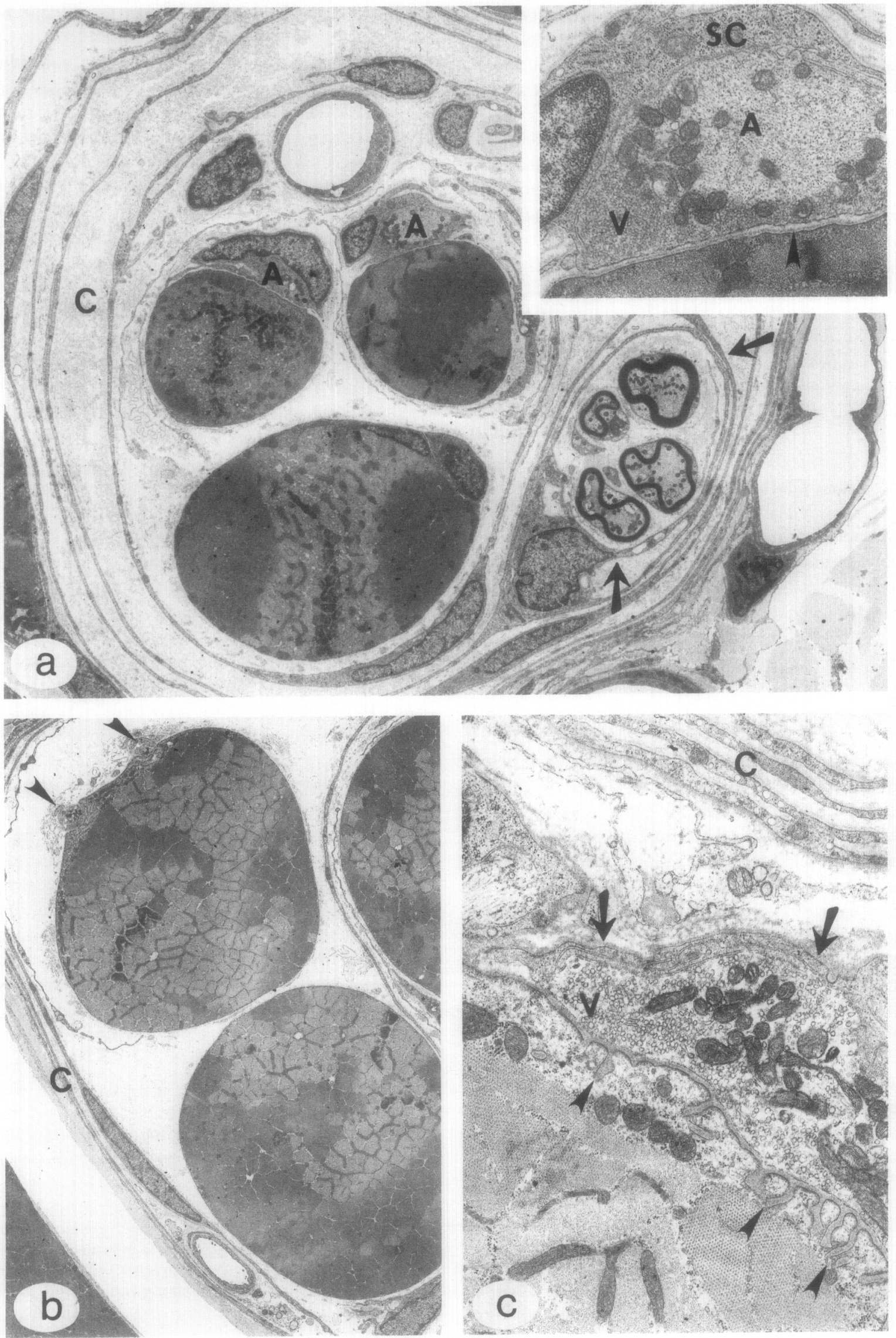
rat spindles (James and Meek, 1971; Merrilees, 1960). Milburn (1976) also observed capillaries in regenerated rat spindles, and Schiaffino and Pierobon Bormioli (1976) found them in spindles that had been denervated and reinnervated during early postnatal development. During either regeneration or reinnervation, the stimulus for sprouting of blood vessels into spindles is not clear. In muscle grafts, regenerating blood vessels often follow preexisting tubes of basal lamina into the graft, although the early ingrowth of the vascular supply may occur independently of this scaffold (Hansen-Smith et al., 1980). Capillaries might enter spindles along sheaths of degenerated nerve fibers or at spindle poles along basal laminae of degenerated intrafusal fibers.

Aside from the small group of specialized muscle fibers in nerve-intact grafts, all other encapsulated fibers were morphologically indistinguishable from the surrounding extrafusal fibers. This was true at both the light and the electron microscopic levels of observation. While encapsulated fibers in nerve-intact grafts were generally smaller than the extrafusal fibers, this size difference was much less apparent in standard grafts, and was often nonexistent in nonreinnervated grafts. Experiments involving denervation of spindles at various stages of development suggest that an increase in the size of intrafusal fibers may be caused by absence of their specific innervation (Hník and Zelená, 1961; Schiaffino and Pierobon Bormioli, 1976; Schröder, 1974a,b; Yellin, 1970). While appropriate innervation may also influence the size of regenerating intrafusal fibers, other variables, such as local vascular supply, might also be critical.

Histochemistry. The ATPase profiles of all regenerated intrafusal muscle fibers was abnormal. Even when more than one fiber type was present within a spindle capsule, the unique ATPase profile of the nuclear bag fibers was absent. The abnormal patterns of ATPase activity very likely reflect varying degrees and patterns of reinnervation of the muscle grafts. Standard reinnervated EDL grafts acquire a mixed population of fiber types (Carlson and Gutmann, 1975) but the distribution of fast and slow fibers is usually abnormal; this seems to result from an abnormal distribution of motor units during reinnervation of the grafts. The fairly uniform ATPase profile of both encapsulated and extrafusal fibers seen in the present study suggest that both types of fiber have been reinnervated by similar neurons.
Uniform staining of intrafusal and extrafusal fibers might also indicate a complete absence of innervation, especially in standard and nonreinnervated grafts. On the other hand, nerve-intact grafts demonstrated a greater heterogeneity of fiber types which may be attributable to an increased efficiency and/or accuracy of reinnervation.

\section{Reinnervation of Regenerated Muscle Spindles}

Both this study and previous reports (Carlson et al., 1979; Rogers and Carlson, 1981) demonstrate reinnervation of regenerated spindles on the basis of silver staining. However, light microscopic analysis cannot provide evidence that nerve fibers form morphologically differentiated junctions with intrafusal muscle fibers or that nerve terminations are sensory or motor in nature. The results of this study demonstrate that both sensory and motor axons may reestablish peripheral connections within spindles, but abnormalities of neurally induced morphological and histochemical characteristics of spindles strongly suggest that such junctions are few and/or faulty. The presence of intact nerve sheaths in nerve-intact grafts is likely to increase the frequency of accurate sensory neuron-muscle connections, just as motor reinnervation of extrafusal muscle fibers is facilitated in these grafts (Carlson et al., 1981). Faulty reinnervation by sensory neurons might be caused by factors other than an inability of these neurons to retrace accurate paths to appropriate targets. Ip et al. (1977) found that when reinnervation of extrafusal muscle by alpha motoneurons was slowed by ventral root section, there were deficiences in sensory reinnervation of spindles. While the mechanisms of this phenomenon are not clear, a similar effect on sensory innervation of regenerating spindles might be related to deficiencies of motor reinnervation of regenerating extrafusal muscle fibers (Carlson et al., 1979). In addition, innervation of regenerating spindles by alpha motoneurons might occur and would explain many of the similarities between regenerated intrafusal and extrafusal muscle fibers.

Although the results of this study strongly suggest inadequacies in sensory innervation of regenerating muscle grafts, a more accurate assessment of its occurrence must be obtained physiologically. Such an analysis is in progress and will provide further information

FIG. 8. (a) Sixty-day nerve-intact graft. Nerve bundle (arrows) within spindle capsule (C) contains both myelinated and nonmyelinated axons. Two nonmyelinated axons (A) are closely associated with the the encapsulated muscle fibers and are covered by Schwann cells. One of these axons is enlarged in the inset. SC, Schwann cell; arrowhead, basal lamina material between axolemma and sarcolemma; V, accumulation of vesicles. $\times 4000$; inset, $\times 18,500$. (b) Two motor terminations (arrowheads) on one encapsulated fiber in a 60-day nerve-intact graft. C, capsule. $\times 2400$. (c) Neuromuscular junction on an encapsulated fiber in a 60-day standard graft. C, capsule; arrows, Schwann cell processes; arrowheads, postjunctional folds; $\mathrm{V}$, vesicles. $\times 15,800$. 
concerning differentiation of regenerating muscle in addition to defining the functional capabilities of regenerated muscle spindles.

I am grateful to Mr. John Beckerman for his expert assistance with the photography and to Dr. Bruce M. Carlson for his support and encouragement throughout this study. This work was supported by grants from the NIH and the Muscular Dystrophy Association of America to Bruce Carlson.

\section{REFERENCES}

BADER, D. (1980). Reinnervation of motor endplate-containing and motor endplateless muscle grafts. Dev. Biol. 77, 315-327.

BENOIT, P. W., and BELT, D. (1970). Destruction and regeneration of skeletal muscle after treatment with a local anesthetic, bupivacaine (Marcaine). J. Anat. 107, 547-556.

BREZEZINSKI, D. K. (1961). Untersuchungen zur Histochemie der Muskelpindeln. II. Mitteilung. Acta Histochem. 12, 227-288.

CARLSON, B. M. (1976). A quantitative study of muscle fiher survival and regeneration in normal, predenervated and Marcaine-treated free muscle grafts in the rat. Exp. Neurol 52, 421-432.

CARLSON, B. M., and GUTMANN, E. (1975). Regeneration in free grafts of normal and denervated muscles in the rat: Morphology and histochemistry. Anat. Rec. 183, 47-82.

CARLSON, B. M., and WAGNER, K. R., and MAX, S. R. (1979). Reinnervation of rat extensor digitorum longus muscles after free grafting. Muscle and Nerve 2, 304-307.

Carlson, B. M., HNik, P., TUCEK, S., VejSADA, R., BADER, D., and FAULKNER, J. A. (1981). Comparison between grafts with intact nerves and standard free grafts of the rat extensor digitorum longus muscle. Physiol. Bohem. 30, 505-513.

Chayen, J., Bitensky, L., and Butcher, R. (1979) "Practical Histochemistry." Wiley, New York.

DeSantis, M., and NoRMaN, W. P. (1979). An ultrastructural study of nerve termial degeneration in muscle spindles of the Tenuissimus muscle of the cat. J. Neurocytol. 8, 67-80.

Elliot, B. J., and HARRIMAN, D. G. F. (1974). Growth of human muscle spindles in vitro. Nature (London) 251, 622-624.

HAKELIUS, L. (1977). Free whole muscle grafting: A survey. In "Reanimation of the Paralyzed Face", (L. Rubin, ed.), pp. 136-154. Mosby, St. Louis.

Hakelius, L. (1979). Free muscle grafting. Clin. Plastic Surg. 6, 301316.

HALL-CRAGGS, E. C. B. (1974). Rapid degeneration and regeneration of a whole skeletal muscle following treatment with bupivacaine (Marcaine). Exp. Neurol. 43, 349-358.

HANSEN-SMiTH, F. M., and CARLSON, B. M. (1979). Cellular responses to free grafting of the extensor digitorum longus muscle of the rat. J. Neurol. Sci. 41, 149-173.

Hansen-Smith, F. M., Carlson, B. M., and Irwin, K. L. (1980). Revascularization of the freely grafted extensor digitorum longus muscle in the rat. Amer. J. Anat. 158, 65-82.

HNIK, P., and ZELENÁ, J. (1961). Atypical spindles in reinnervated rat muscles. J. Embryol. Exp. Morphol. 9, 456-467.

HsU, L. (1974). The role of nerves in the regeneration of minced skeletal muscle in adult anurans. Anat. Rec. 179, 119-136.

IP, M. C., VRbova, G., and WestBuRY, D. R. (1977). The sensory reinnervation of hind limb muscles of the cat following denervation and de-efferentation. Neuroscience 2, 423-434.
JAMES, N. T. (1971). The histochemical demonstration of mucopolysaccharide in the lymph space of muscle spindles. J. Anat. 110, 165. JAMES, N. T., and MEEK, G. A. (1971). The blood supply of rat muscle spindles. $J$. Anat. 110, 165.

KUCERA, J. (1980a). Abnormal muscle spindles in the mature free autograft of the rat skeletal muscle. Neurology 30, 405.

KUCERA, J. (1980b). Myofibrillar ATPase activity of intrafusal fibers in chronically de-afferented rat muscle spindles. Histochemistry 66 , 221-228.

KuCERA, J., Dorovini-ZIS, K., and ENGEL, W. K. (1978). Histochemistry of rat intrafusal muscle fibers and their motor innervation. $J$. Histochem. Cytochem. 25, 1260-1263.

Libelius, R., Sonesson, B., Stamenovic, B. A., and Thesleff, S. (1970). Denervation-like changes in skeletal muscle after treatment with a local anesthetic (Marcaine). J. Anat. 106, 297-309.

MaCkenson-Dean, C. A., Hikida, R. S., and Frangowlakis, T. M. (1981). Formation of muscle spindles in regenerated avian muscle grafts. Cell Tissue Res. 217, 37-41.

MerRILEes, N. C. R. (1960). The fine structure of muscle spindles in the lumbrical muscles of the rat. J. Biophys. Biochem. Cytol. 7, 725742 .

MilBuRn, A. (1973). The early development of muscle spindles in the rat. J. Cell Sci. 12, 175-195.

MILBURN, A. (1976). The effect of the local anesthetic bupivacaine on the muscle spindle of the rat. J. Neurocytol. 5, 425-446.

Mufti, S. A., Carlson, B. M., Maxwell, L. C., and Faulkner, J. A. (1977). Free autografting of entire limb muscles in the cat: morphology. Anat. Rec. 188, 417-430.

ONTELL, M., and FENG, K. C. (1981). The three-dimensional cytoarchitecture and pattern of motor innervation of branched striated myotubes. Anat. Rec. 200, 11-31.

OvALLE, W. K. (1971). Fine structure of rat intrafusal muscle fibers: The polar region. J. Cell Biol. 51, 83-103.

OVALLE, W. K. (1972a). Fine structure of rat intrafusal muscle fibers: The equatorial region. J. Cell Biol. 52, 382-396.

OVALLE, W. K. (1972b). Motor nerve terminals on rat intrafusal muscle fibers, a correlated light and electron microscopic study. J. Anat. 111, 239-252.

Rogers, S. L., and CARLson, B. M. (1981). A quantitative assessment of muscle spindle formation in reinnervated and non-reinnervated grafts of the rat extensor digitorum longus muscle. Neuroscience 6 , 87-94.

Schiaffino, S., and Pierobon, Bormioli, S. (1976). Morphogenesis of rat muscle spindle formation after nerve lesion during early postnatal development. J. Neurocytol. 5, 319-336.

SchmalbRuch, H. (1977) Regeneration of soleus muscle of rat autografted in toto as studied by electron microscopy. Cell Tiss. Res. 177, 159-180.

SCHRÖDER, J. M. (1974a). The fine structure of de- and reinnervated muscle spindles. I. The increase, atrophy, and hypertrophy of intrafusal muscle fibers. Acta Neurupathol (Berl.) 30, 109-128.

SCHRÖDER, J. M. (1974b). The fine structure of de- and reinnervated muscle spindles. II. Regenerated sensory and motor nerve terminals. Acta Neuropathol. 30, 129-144.

Schröder, J. M., Kemme, P. T., and SCholz, L. (1979). The fine structure of denervated and reinnervated muscle spindles: Morphometric study of intrafusal muscle fibers. Acta Neuropathol. 46, 95106.

SNow, M. H. (1978). An autoradiographic study of satellite cell differentiation into regenerating myotubes following transplantation of muscles in young rats. Cell Tissue Res. 186, 535-540. 
THOMPSON, N. (1979). Free muscle graft pharyngoplasty with palatal retroposition in treatment of velopharyngeal incompetence. Chirugia Plastica 5, 1-14.

Tower, S. (1932). Atrophy and degeneration in the muscle spindle. Brain 55, 77-90.

Trupin, G. L., Hsu, L., and HsiEH, Y-H. (1979). Satellite cell mimics in regenerating skeletal. In "Muscle Regeneration" (A. Mauro, ed). Raven Press, New York.

VRACKo, R., and BENDITT, E. P. (1972). Basal lamina: The scaffold for orderly cell replacement. J. Cell Biol. 55, 406-419.

WERNER, J, K. (1973). Duration of normal innervation required for complete differentiation of muscle spindles in newborn rats. Exp. Neurol. 41, 214-217.
YELLLIN, H. (1970). Evidence favoring neural regulation of intrafusal muscle fiber metabolism in the neonatal rat. Anat. Rec. 166, 400. ZFI.FNÁ, J. (1957). The morphogenetic influence of innervation on the ontogenetic development of muscle spindles. J. Embryol. Exp. Morphol. 5, 183-292.

ZELENÁ, J., and SoUkUP, T. (1973). Development of muscle spindles deprived of fusimotor innervation. $Z$. Zellforsch. 144, 435-452.

ZELENÁ, J., and SOUKUP, T. (1974). The differentiation of intrafusal fiber types in rat muscle spindles after motor denervation. Cell Tissue Res. 153, 115-136.

ZELENÄ, J., and SOUKUP, T. (1975). Ultrastructural changes of developing muscle spindles after denervation. Folia Morphol. 23, 275277. 